\title{
Opinión de futuros equipos docentes de educación primaria sobre la implementación del mobile learning en el aula
}

\author{
Opinion of Future Primary Education Teachers on the Implementation of \\ Mobile Learning in the Classroom
}

\section{Opinião das futuras equipas de ensino do ensino primário sobre a implementação da aprendizagem móvel na sala de aula}

Francisco Javier Hinojo-Lucena

Universidad de Granada

Granada, España

fhinojo@ugr.es

https://orcid.org/0000-0002-9507-4058

Inmaculada Aznar-Díaz

Universidad de Granada

Granada, España

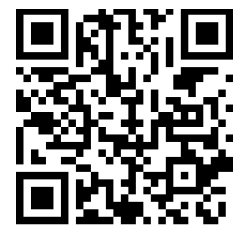

iaznar@ugr.es

https://orcid.org/0000-0002-0018-1150

María Pilar Cáceres-Reche

Universidad de Granada

Granada, España

caceres@ugr.es

https://orcid.org/0000-0002-6323-8054

José María Romero-Rodríguez

Universidad de Granada

Granada, España

romejo@ugr.es

https://orcid.org/0000-0002-9284-8919

Recibido • Received • Recebido: 11 / 06 / 2018

Corregido • Revised • Revisado: 28 / 04 / 2019

Aceptado • Accepted • Aprovado: 25/ 06 / 2019

Resumen: Las tecnologías de la información y comunicación (TIC) son una tendencia a implementar en el ámbito educativo. En concreto, la universalización en el uso de los dispositivos móviles ha posibilitado su aplicación en diferentes etapas educativas. El objetivo de este trabajo ha sido conocer la opinión de futuros equipos docentes de educación primaria sobre la implementación del mobile learning en el aula. La metodología empleada es de corte cuantitativo, con la finalidad de describir la realidad observada en una muestra de estudiantado universitario del grado en 
doi: http://dx.doi.org/10.15359/ree.23-3.14

URL: http://www.una.ac.cr/educare

CORREO: educare@una.cr

Educación Primaria de la Universidad de Granada. Así mismo, se utilizó como instrumento de recogida de datos un cuestionario elaborado ad hoc, con base en una escala Likert de 4 niveles. El análisis de los datos se ha realizado mediante el software de análisis estadístico SPSS y AMOS. Entre los resultados se resalta la percepción positiva del estudiantado sobre la incorporación de la metodología mobile learning en educación primaria, así como la adecuación en el uso de tecnologías móviles emergentes como la realidad aumentada y realidad virtual. Por último, se discuten distintas implicaciones y recomendaciones sobre la aplicación de los dispositivos móviles en el aula, entre ellas la generalización de estos recursos en este espacio, reticencias que todavía existen por parte del personal docente (maestros y maestras) y la mejora del aprendizaje estudiantil.

Palabras claves: Mobile learning; dispositivos móviles; educación primaria; TIC.

Abstract: Information and Communication Technologies (ICT) are a trend implemented in the educational field. In particular, the universalization in the use of mobile devices has made possible its application in different educational stages. The purpose of this paper has been to know the opinion of the future teachers of Primary Education about the implementation of mobile learning in the classroom. A quantitative design methodology was used to describe the reality observed in a sample of university students of the degree in Primary Education of the University of Granada. Likewise, a questionnaire prepared ad hoc was used as a data collection instrument, based on a Likert scale of 4 levels. The analysis of the data has been made using the statistical analysis software SPSS and AMOS. Among the results, the positive perception of the students on the incorporation of the mobile learning methodology in Primary Education is highlighted, as well as the adequacy in the use of emerging mobile technologies such as Augmented Reality and Virtual Reality. Finally, different implications and recommendations on the application of mobile devices in the classroom are discussed, including the generalization of these resources in the classroom, reticence that still exists on the part of some teachers and the improvement of student learning.

Keywords: Mobile learning; mobile devices; primary education; ICT.

Resumo: As tecnologias da informação e da comunicação (TIC) são uma tendência a implementar no domínio da educação. Especificamente, a universalização do uso de dispositivos móveis tornou possível a sua aplicação em diferentes etapas educativas. O objetivo deste trabalho foi conhecer a opinião das futuras equipes de ensino do ensino fundamental sobre a implementação da aprendizagem móvel em sala de aula. A metodologia utilizada é quantitativa, com o objetivo de descrever a realidade observada em uma amostra de estudantes universitários do ensino fundamental da Universidade de Granada. De igual modo, foi utilizado como instrumento de recolha de dados um questionário ad hoc baseado numa escala de Likert de 4 níveis. A análise dos dados foi realizada utilizando o software de análise estatística SPSS e AMOS. Entre os resultados, destaca-se a percepção positiva dos estudantes sobre a incorporação da metodologia de aprendizagem móvel no ensino fundamental, bem como a adaptação no uso de tecnologias móveis emergentes, como realidade aumentada e realidade virtual. Finalmente, são discutidas diferentes implicações e recomendações sobre a aplicação de dispositivos móveis na sala de aula, incluindo a generalização desses recursos nesse espaço, as reticências que ainda existem por parte do corpo docente (professores) e a melhoria da aprendizagem dos alunos.

Palavras-chave: Aprendizagem móvel; dispositivos móveis; educação primária; TIC. 


\section{Introducción}

Los dispositivos móviles son un recurso clave para la enseñanza en la educación del siglo XXI. En esta etapa histórica, totalmente digitalizada, se prioriza la actualización y renovación de la enseñanza a través de las tecnologías emergentes. En este caso, la tecnología móvil ofrece grandes posibilidades para la mejora del aprendizaje (Chaves-Barboza y Sola-Martínez, 2018), al mismo tiempo que aumenta la motivación del estudiantado (Cuesta y Mañas, 2016; Fombona y Pascual, 2013), favorece el trabajo cooperativo (Nguyen, Barton y Nguyen, 2015; Monguillot, González, Guitert y Zurita, 2014) y permite el desarrollo de competencias digitales (GómezGonzalvo, Atienza y Mir, 2015).

En concreto, la implementación de los dispositivos móviles en la educación recibe el nombre de mobile learning o aprendizaje móvil, definido por Brazuelo y Gallego (2011, p. 17) como "la modalidad educativa que facilita la construcción del conocimiento, la resolución de problemas de aprendizaje y el desarrollo de destrezas o habilidades diversas de forma autónoma y ubicua gracias a la mediación de dispositivos móviles portátiles".

A su vez, los recursos limitados de los que disponen los centros educativos requieren de fórmulas alternativas para la aplicación de una metodología basada en el aprendizaje móvil. Se opta, así, por la posibilidad de que el estudiantado traiga su propio dispositivo al aula. Este concepto se denomina Bring Your Own Devices (BYOD) y se vincula a la introducción real de esta metodología docente (Suárez-Guerrero, Lloret-Catalá y Mengual-Andrés, 2016).

La efectividad de los dispositivos móviles para el aprendizaje radica en su característica principal, la ubicuidad, la cual hace posible utilizar el dispositivo en cualquier momento y lugar debido a sus prestaciones (Vázquez-Cano, 2015). Así mismo, aunque algunos estudios tildan el uso de dispositivos móviles como elementos de distracción en el aula (Cantillo, Roura y Sánchez, 2012; Gértrudix y Barroso-Megías, 2016), no cabe duda de sus beneficios (Wu et al., 2012). Estos beneficios se alcanzan con el buen uso de los dispositivos móviles, aplicándolos en un espacio determinado y durante un tiempo previamente establecido.

Además, los dispositivos móviles permiten la utilización de diferentes herramientas digitales como los códigos QR (Romero y Rodríguez-García, 2017), la realidad aumentada (Cabero, Fernández y Marín, 2017), Realidad Virtual (Aznar-Díaz, Romero-Rodríguez y RodríguezGarcía, 2018) y la impresión 3D (Saorin et al., 2016), sin duda una poderosa herramienta para el aprendizaje como destacan estas investigaciones.

Por otro lado, estudios previos sobre la percepción del estudiantado acerca de la metodología mobile learning recogen una opinión positiva de su uso para la mejora del aprendizaje (Contreras, 2010; Garay, Tejada y Castaño, 2016; Grané, Crescenzi y Olmedo, 2013; Rivera, Sánchez, Romo, Jaramillo y Valencia, 2013). 
doi: http://dx.doi.org/10.15359/ree.23-3.14

URL: http://www.una.ac.cr/educare

CORREO: educare@una.cr

A nivel mundial, el informe Horizon, referente en tecnología educativa, indica en su edición de 2017 que el mobile learning es una realidad a incorporar en el sistema educativo de forma generalizada, en un periodo temporal a corto plazo (Adams et al., 2017).

Teniendo en cuenta estas consideraciones y la relevancia actual de la temática, en este trabajo se planteó como objetivo conocer la opinión de futuros maestros y futuras maestras de educación primaria sobre la implementación de la metodología mobile learning en el aula.

\section{Metodología}

En este estudio se ha seguido una metodología de corte cuantitativo con la finalidad de describir la realidad observada (Grané, Fernández y Baptista, 2013). El instrumento utilizado para la recogida de información ha sido el cuestionario, compuesto por 20 ítems y elaborado ad hoc en base a las consideraciones de distintos estudios sobre el mobile learning (Aznar-Díaz, Cáceres-Reche y Romero-Rodríguez, 2018; Cabero et al., 2017; Fombona y Pascual, 2013; Garay et al., 2016; Rivera et al., 2013).

Las opciones de respuesta se recogieron en una escala Likert de cuatro niveles: 4 - Totalmente de acuerdo; 3 - De acuerdo; 2 - En desacuerdo y; 1 - Totalmente en desacuerdo. El análisis de fiabilidad del cuestionario presenta unos valores aceptables en la prueba alfa de Cronbach $(a=, 918)$.

Para la conformación de la muestra objeto de estudio, se ha utilizado un muestreo criterial, estableciendo como criterio principal la disponibilidad de sus participantes, a quienes se les invitó a participar a partir del envío de un correo electrónico con el enlace al cuestionario. La población se ha compuesto por 591 estudiantes de universidad de segundo curso del grado en Educación Primaria de la Universidad de Granada, con matrícula durante el curso 20172018. Finalmente, se determinó un tamaño muestral significativo $(n=132)$ con un intervalo de confianza del $95 \%$ y un margen de error del 7,5\%. Este grupo se compone por un total de 92 mujeres $(69,7 \%)$ y 40 hombres (30,3\%), con edades comprendidas entre 18-20 (60,6 \%), 21-23 $(24,2 \%), 24-26$ (3 \%), 27-29 (3 \%) y más de 30 años (9,1 \%). Además, 36 (27,3\%) manifiestan haber cursado alguna materia donde se ha implementado la metodología mobile learning, frente a 96 que no la han experimentado nunca $(72,7 \%)$.

El análisis factorial del cuestionario muestra que el $72,182 \%$ de la varianza es explicada por los cinco primeros ítems: Ítem 1. Considero que para poder desarrollar realmente una metodología basada en el mobile learning cada estudiante tendría que traer su propio dispositivo móvil al aula; Ítem 2. Considero una ventaja la posibilidad de utilizar el dispositivo móvil en cualquier momento y lugar para que el alumnado acceda a la información; Ítem 3. Considero que los dispositivos móviles aplicados en el aula son facilitadores del aprendizaje más que distractores; Ítem 4. El desarrollo de actividades con dispositivos móviles en el aula puede disminuir su uso inadecuado en la escuela; Ítem 5. La posibilidad de utilizar cualquier aplicación móvil (app) ha- 
cen que el mobile learning sea un potente recurso para el aprendizaje (ver Tabla 1). Así mismo, la medida KMO de adecuación del muestreo recoge una puntuación de ,697 y la prueba de esfericidad de Bartlett un chi-cuadrado aproximado de 2086,310, 190 gl con significación a ,000.

Tabla 1: Varianza total explicada del cuestionario utilizado para la recogida de datos, método de componentes principales con rotación varimax

\begin{tabular}{|c|c|c|c|c|c|c|c|c|c|}
\hline \multirow[b]{2}{*}{ Ítems } & \multicolumn{3}{|c|}{ Autovalores } & \multicolumn{3}{|c|}{$\begin{array}{l}\text { Sumas de las saturaciones al } \\
\text { cuadrado de la extracción }\end{array}$} & \multicolumn{3}{|c|}{$\begin{array}{l}\text { Sumas de las saturaciones al } \\
\text { cuadrado de la rotación }\end{array}$} \\
\hline & Total & $\begin{array}{c}\% \\
\text { varianza }\end{array}$ & $\begin{array}{c}\% \\
\text { acumulado }\end{array}$ & Total & $\begin{array}{c}\% \\
\text { varianza }\end{array}$ & $\begin{array}{c}\% \\
\text { acumulado }\end{array}$ & Total & $\begin{array}{c}\% \\
\text { varianza }\end{array}$ & $\begin{array}{c}\% \\
\text { acumulado }\end{array}$ \\
\hline 1 & 8,239 & 41,194 & 41,194 & 8,239 & 41,194 & 41,194 & 3,753 & 18,767 & 18,767 \\
\hline 2 & 1,841 & 9,205 & 50,399 & 1,841 & 9,205 & 50,399 & 2,924 & 14,622 & 33,389 \\
\hline 3 & 1,676 & 8,381 & 58,780 & 1,676 & 8,381 & 58,780 & 2,782 & 13,911 & 47,300 \\
\hline 4 & 1,483 & 7,414 & 66,193 & 1,483 & 7,414 & 66,193 & 2,630 & 13,151 & 60,451 \\
\hline 5 & 1,198 & 5,989 & 72,182 & 1,198 & 5,989 & 72,182 & 2,346 & 11,731 & 72,182 \\
\hline 6 & 927 & 4,636 & 76,818 & & & & & & \\
\hline 7 & ,802 & 4,011 & 80,828 & & & & & & \\
\hline 8 & 731 & 3,654 & 84,482 & & & & & & \\
\hline 9 & ,632 & 3,159 & 87,641 & & & & & & \\
\hline 10 &, 524 & 2,620 & 90,262 & & & & & & \\
\hline 11 & ,423 & 2,116 & 92,378 & & & & & & \\
\hline 12 & ,324 & 1,620 & 93,998 & & & & & & \\
\hline 13 & 295 & 1,473 & 95,471 & & & & & & \\
\hline 14 & ,256 & 1,278 & 96,749 & & & & & & \\
\hline 15 & ,214 & 1,071 & 97,820 & & & & & & \\
\hline 16 & 151 & 756 & 98,576 & & & & & & \\
\hline 17 & ,113 & ,564 & 99,140 & & & & & & \\
\hline 18 & ,080 & ,401 & 99,541 & & & & & & \\
\hline 19 & ,061 & ,305 & 99,847 & & & & & & \\
\hline 20 & ,031 & 153 & 100,000 & & & & & & \\
\hline
\end{tabular}

Nota: Elaboración propia, a partir del programa de análisis estadístico SPSS. 
doi: http://dx.doi.org/10.15359/ree.23-3.14

URL: http://www.una.ac.cr/educare

CORREO: educare@una.cr

Por otro lado, se ha procedido a realizar un análisis factorial confirmatorio con tres mediciones para verificar la correlación entre las variables latentes "percepción positiva sobre el uso de los dispositivos móviles", "implementación de tecnologías móviles emergentes" y "desarrollo de habilidades a partir del mobile learning". Para ello, se ha ejemplificado gráficamente la correlación existente a través del modelo de ecuaciones estructurales (Ruiz, Pardo y San Martín, 2010).

Cabe mencionar que el análisis de los datos se ha llevado a cabo a través de los programas de análisis estadístico SPSS y AMOS en su versión 24.

\section{Resultados, análisis y discusión}

Los ítems del cuestionario se han agrupado en distintas dimensiones, correspondientes a: percepción positiva sobre el uso de los dispositivos móviles (ítems 1-4); implementación de tecnologías emergentes (ítems 5-9); desarrollo de habilidades a partir del mobile learning (ítems 10-15) y perspectiva como docente (ítems 16-20).

Si atendemos las respuestas con base en la escala Likert, por cada pregunta se obtuvieron los siguientes puntajes:

Ítem 1.Considero que para poder desarrollar realmente una metodología basada en el mobile learning cada estudiante tendría que traer su propio dispositivo móvil al aula, las contestaciones se distribuyeron en desacuerdo $(15,2 \%)$, de acuerdo $(63,6 \%)$ y totalmente de acuerdo $(21,2 \%)$.

Ítem 2. Considero una ventaja la posibilidad de utilizar el dispositivo móvil en cualquier momento y lugar para que el alumnado acceda a la información: totalmente en desacuerdo ( 3 $\%)$, en desacuerdo ( $27,3 \%)$, de acuerdo (33,3 \%) y totalmente de acuerdo (36,4 \%).

Ítem 3. Considero que los dispositivos móviles aplicados en el aula son facilitadores del aprendizaje más que distractores: totalmente en desacuerdo $(21,2 \%)$, en desacuerdo $(48,5 \%)$ y de acuerdo (30,3\%).

Ítem 4. El desarrollo de actividades con dispositivos móviles en el aula puede disminuir su uso inadecuado en la escuela: totalmente en desacuerdo (6,1\%), en desacuerdo $(27,3 \%)$, de acuerdo (48,5\%) y totalmente de acuerdo (18,2\%).

Ítem 5. La posibilidad de utilizar cualquier aplicación móvil (app) hace que el mobile learning sea un potente recurso para el aprendizaje: totalmente en desacuerdo $(6,1 \%)$, en desacuerdo $(12,1 \%)$, de acuerdo $(63,6 \%)$ y totalmente de acuerdo $(18,2 \%)$.

Ítem 6. Las actividades basadas en la creación y lectura de códigos $Q R$ son un buen recurso para implementar en la etapa de educación primaria: totalmente en desacuerdo $(6,1 \%)$, en desacuerdo (30,3\%), de acuerdo (51,5\%) y totalmente de acuerdo $(12,1 \%)$. 
Ítem 7. Las actividades basadas en la realidad aumentada son un buen recurso para implementar en la etapa de educación primaria: en desacuerdo $(9,1 \%)$, de acuerdo $(42,4 \%)$ y totalmente de acuerdo (48,5\%).

Ítem 8. Las actividades basadas en la realidad virtual son un buen recurso para implementar en la etapa de educación primaria: en desacuerdo (30,3\%), de acuerdo (30,3\%) y totalmente de acuerdo (39,4\%).

Ítem 9. Las actividades basadas en el uso de apps de diseño y modelado en 3D para su posterior impresión (en una impresora 3D) son un buen recurso para implementar en la etapa educación primaria: en desacuerdo (27,3\%), de acuerdo (39,4\%) y totalmente de acuerdo (33,3\%).

Ítem 10. Considero que el mobile learning puede incidir en la mejora del aprendizaje del alumnado: totalmente en desacuerdo (3\%), en desacuerdo (24,2\%), de acuerdo (54,5\%) y totalmente de acuerdo (18,2\%).

Ítem 11. El hecho de introducir el dispositivo móvil en el aula hace que mejore la motivación del estudiantado: totalmente en desacuerdo (3\%), en desacuerdo $(21,2 \%)$, de acuerdo $(36,4 \%)$ y totalmente de acuerdo $(39,4 \%)$.

Ítem 12. Atendiendo las diversas actividades que pueden realizarse con los dispositivos móviles en el aula y fuera de ella, considero que el mobile learning favorece el trabajo cooperativo: totalmente en desacuerdo (9,1\%), en desacuerdo (42,4\%), de acuerdo (39,4\%) y totalmente de acuerdo (9,1\%).

Ítem 13. La participación del alumnado sería mayor respecto a una metodología tradicional si se habilitan grupos de trabajo en la red, a través de plataformas virtuales, redes sociales o apps como WhatsApp: totalmente en desacuerdo (9,1\%), en desacuerdo (24,2\%), de acuerdo $(48,5 \%)$ y totalmente de acuerdo $(18,2 \%)$.

Ítem 14. Aprender en edades tempranas a utilizar adecuadamente y dar un buen uso al dispositivo móvil puede ser una medida para evitar el ciberbullying: totalmente en desacuerdo $(9,1 \%)$, en desacuerdo (18,2\%), de acuerdo (30,3\%) y totalmente de acuerdo $(42,4 \%)$.

Ítem 15. Considero que la metodología mobile learning puede contribuir al desarrollo de competencias digitales en el alumnado: en desacuerdo $(9,1 \%)$, de acuerdo $(54,5 \%)$ y totalmente de acuerdo (36,4\%).

Ítem 16. Creo apropiada la introducción de los dispositivos móviles como metodología docente en la etapa de educación primaria: totalmente en desacuerdo $(9,1 \%)$, en desacuerdo $(24,2 \%)$, de acuerdo (54,5\%) y totalmente de acuerdo (12,1\%).

Ítem 17. Considero que es necesaria la combinación del mobile learning con la metodología tradicional (clase magistral, libros de texto...): totalmente en desacuerdo (3\%), en desacuerdo $(15,2 \%)$, de acuerdo (51,5\%) y totalmente de acuerdo (30,3\%). 
doi: http://dx.doi.org/10.15359/ree.23-3.14

URL: http://www.una.ac.cr/educare

CORREO: educare@una.cr

Ítem 18. Considero que el personal docente debe tener conocimientos tecnológicos para aplicar el mobile learning en el aula: en desacuerdo (3\%), de acuerdo (18,2\%) y totalmente de acuerdo $(78,8 \%)$.

Ítem 19. Creo que la metodología mobile learning se implementará de forma general en educación primaria en un corto periodo de tiempo ( 1 - 2 años): totalmente en desacuerdo $(12,1$ $\%)$, en desacuerdo (48,5\%), de acuerdo (33,3\%) y totalmente de acuerdo (6,1 \%).

Ítem 20. Aplicaría la metodología mobile learning como futuro personal docente en mi aula: totalmente en desacuerdo $(9,1 \%)$, en desacuerdo $(24,2 \%)$, de acuerdo $(42,4 \%)$ y totalmente de acuerdo (24,2\%).

A modo de síntesis se recogen estos porcentajes en la Tabla 2.

Tabla 2: Porcentajes de las respuestas obtenidas por ítem

\begin{tabular}{ccccc}
\hline Ítem & Totalmente de desacuerdo & En desacuerdo & De acuerdo & Totalmente de acuerdo \\
\hline 1 & 0 & 15,2 & 63,6 & 21,2 \\
2 & 3 & 27,3 & 33,3 & 36,4 \\
3 & 21,2 & 48,5 & 30,3 & 0 \\
4 & 6,1 & 27,3 & 48,5 & 18,2 \\
5 & 6,1 & 12,1 & 63,3 & 18,2 \\
6 & 6,1 & 30,3 & 51,5 & 12,1 \\
7 & 0 & 9,1 & 42,4 & 48,5 \\
8 & 0 & 30,3 & 30,3 & 39,4 \\
9 & 0 & 27,3 & 39,4 & 33,3 \\
10 & 3 & 24,2 & 54,4 & 18,2 \\
11 & 3 & 21,2 & 36,4 & 39,4 \\
12 & 9,1 & 42,4 & 39,4 & 9,1 \\
13 & 9,1 & 24,2 & 48,5 & 18,2 \\
14 & 9,1 & 18,2 & 30,3 & 42,4 \\
15 & 0 & 9,1 & 54,5 & 36,4 \\
16 & 9,1 & 24,2 & 54,5 & 12,1 \\
17 & 3 & 15,2 & 51,5 & 30,3 \\
18 & 0 & 3 & 18,2 & 78,8 \\
19 & 12,1 & 48,5 & 33,3 & 6,1 \\
20 & 9,1 & 24,2 & 42,4 & 24,2 \\
\hline
\end{tabular}

Nota: Elaboración propia. 
A continuación se recogen las medidas de tendencia central y dispersión de cada una de las respuestas, donde se especifica la media, mediana, moda y desviación típica (ver Tabla 3). Al igual, la Figura 1 muestra de modo gráfico las medias por ítem.

Tabla 3: Medidas de tendencia central y dispersión de las respuestas obtenidas

\begin{tabular}{|c|c|c|c|c|}
\hline Ítem & Media & Mediana & Moda & Desviación típica \\
\hline 1 & 3,06 & 3 & 3 & 602 \\
\hline 2 & 3,03 & 3 & 4 & 873 \\
\hline 3 & 2,09 & 2 & 2 & 715 \\
\hline 4 & 2,79 & 3 & 3 & 811 \\
\hline 5 & 2,94 & 3 & 3 & 739 \\
\hline 6 & 2,70 & 3 & 3 & ,761 \\
\hline 7 & 3,39 & 3 & 4 & ,651 \\
\hline 8 & 3,09 & 3 & 4 & ,833 \\
\hline 9 & 3,06 & 3 & 3 & 779 \\
\hline 10 & 2,88 & 3 & 3 & ,731 \\
\hline 11 & 3,12 & 3 & 4 & ,847 \\
\hline 12 & 2,48 & 2 & 2 & 786 \\
\hline 13 & 2,76 & 3 & 3 & ,857 \\
\hline 14 & 3,06 & 3 & 4 & 987 \\
\hline 15 & 3,27 & 3 & 3 & 619 \\
\hline 16 & 2,70 & 3 & 3 & ,800 \\
\hline 17 & 3,09 & 3 & 3 & 756 \\
\hline 18 & 3,76 & 4 & 4 & 496 \\
\hline 19 & 2,33 & 2 & 2 & 768 \\
\hline 20 & 2,82 & 3 & 3 & 906 \\
\hline
\end{tabular}

Nota: Elaboración propia. 
doi: http://dx.doi.org/10.15359/ree.23-3.14

URL: http://www.una.ac.cr/educare

CORREO: educare@una.cr

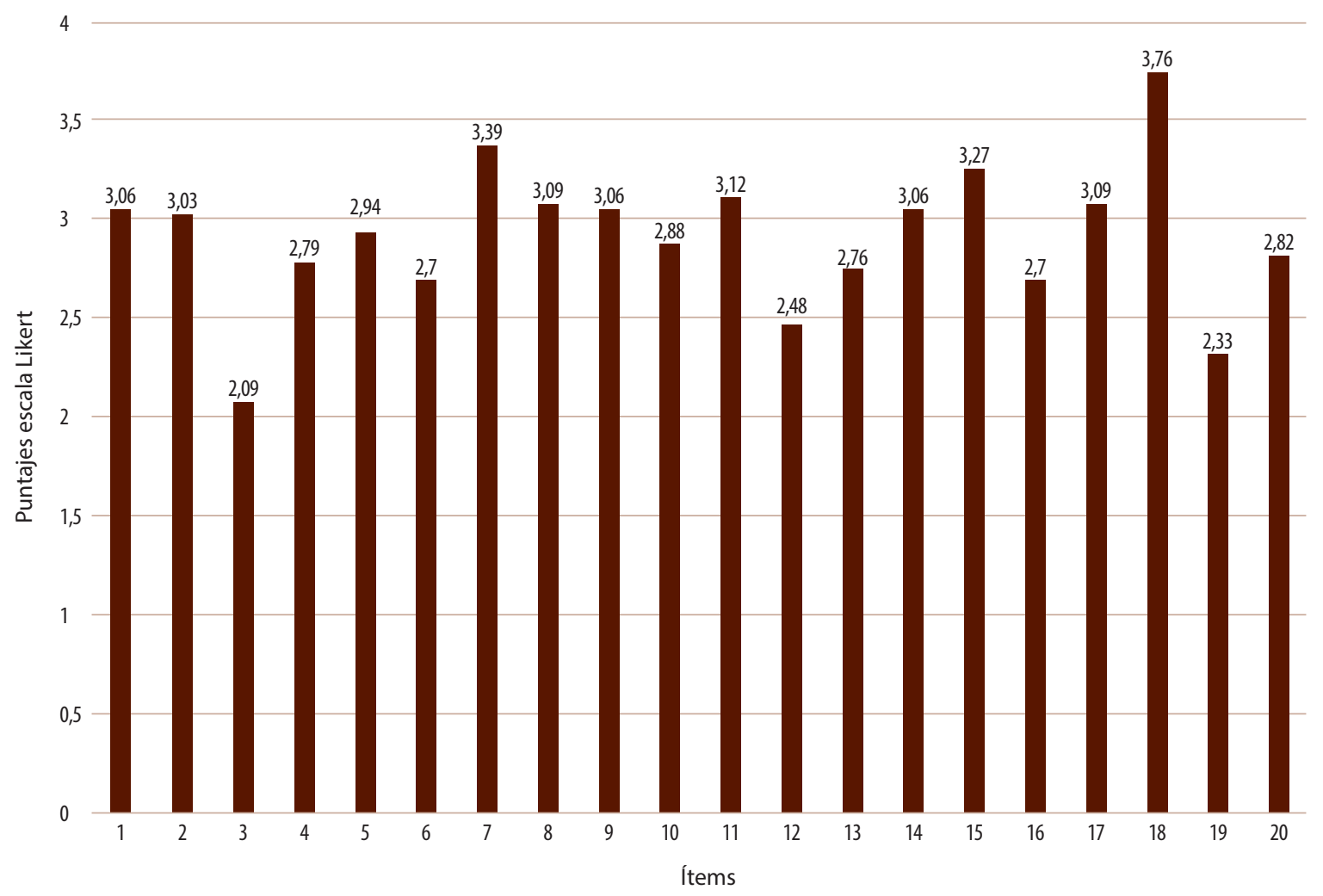

Figura 1: Medias de los ítems con base en la escala Likert.

Nota: Elaboración propia.

Con el análisis factorial confirmatorio se ha verificado la correlación existente entre las dimensiones "percepción positiva sobre el uso de los dispositivos móviles (PDM)", "implementación de tecnologías móviles emergentes (ITE)" y "desarrollo de habilidades a partir del mobile learning (HML)". Además, se ha optado por incluir el ítem 20 (APLI) como variable externa para comprobar la correlación entre la opinión de que los dispositivos móviles favorecen el desarrollo de habilidades y el hecho de implementar el mobile learning en un futuro como docente de primaria.

Los datos muestran que la covarianza es positiva entre las distintas dimensiones (cov >0). De modo que hay una interdependencia positiva entre las dimensiones, lo cual indica que si una de ellas sube en puntuación la otra también lo hará. Por su parte, debido a que la relación crítica (CR) es superior a 1,96 puntos se establece la significación a $<=, 05$, siendo estadísticamente significativas las relaciones entre las distintas dimensiones (ver Tabla 4). 
Tabla 4: Covarianzas y correlaciones entre las distintas competencias

\begin{tabular}{cccccc}
\hline Relación & Covarianza & SE & CR & P & R \\
\hline PDM <--> ITE &, 105 &, 027 & 3,917 & $* * *$ &, 712 \\
PDM <--> HML &, 078 &, 021 & 3,694 & $* * *$ &, 890 \\
ITE <--> HML &, 120 &, 029 & 4,184 & $* * *$ &, 773 \\
HML --> APLI & 2,465 &, 424 & 5,821 & $* * *$ & 1 \\
\hline
\end{tabular}

$\mathrm{CR}=$ relación crítica; $\mathrm{SE}=$ error estándar. $\mathrm{P} * * *$ significación $<, 001$.

Nota: Elaboración propia.

Por otro lado, las medidas de bondad de ajuste absoluto indican la exactitud de los datos del modelo (Escobedo, Hernández, Estebané y Martínez, 2016), Se obtuvieron, en este caso, unas medidas aceptables donde el $60 \%$ de la covarianza es reproducida por este (ver Tabla 5).

Tabla 5: Medidas de bondad de ajuste del modelo de ecuación estructural

\begin{tabular}{lcc}
\hline Valor & Obtenido & Esperado \\
RMSEA &, 209 & $<, 05$ \\
RMR &, 068 & Cercano a 0 \\
NNFI &, 534 & $>, 90$ \\
CFI &, 607 & $>, 90$ \\
PGFI &, 489 & Entre $, 5 \mathrm{y}, 7$ \\
\hline
\end{tabular}

Nota: Elaboración propia.

Respecto al modelo de ecuación estructural (ver Figura 2), la primera dimensión (PDM) se define por 4 ítems observables con valores de regresión entre el ,29y ,84. La segunda dimensión (ITE) es definida por 5 variables observables, con valores entre ,55 y,83. Por último, la tercera dimensión (HML) recoge 6 ítems entre 49 y, 90. 
doi: http://dx.doi.org/10.15359/ree.23-3.14

URL: http://www.una.ac.cr/educare

CORREO: educare@una.cr

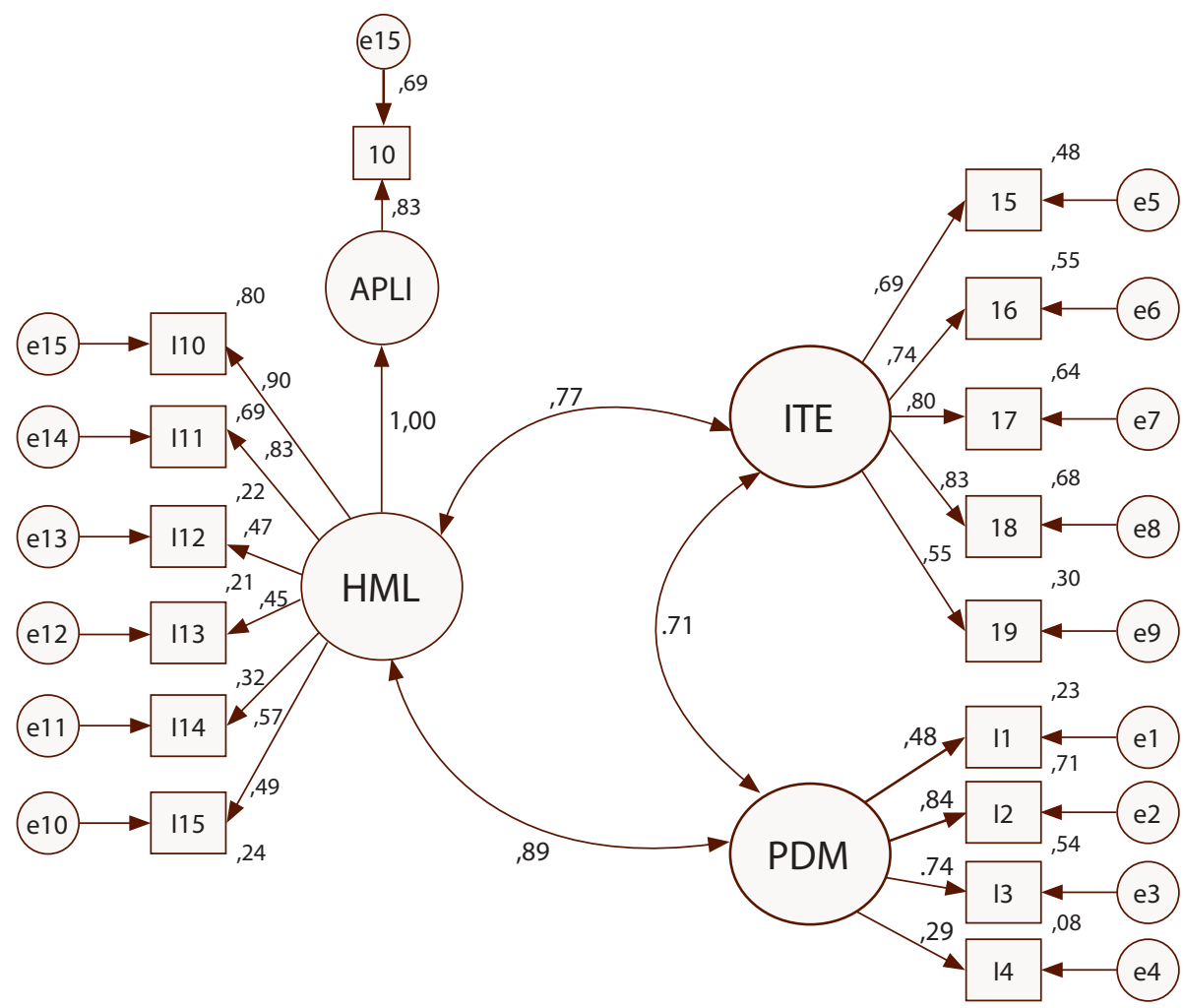

Chi-cuadrado $=678,890, \mathrm{df}=101$, P-value $=, 000$

Figura 2: Ecuación estructural del análisis factorial confirmatorio entre las variables latentes correspondientes a "percepción positiva sobre el uso de los dispositivos móviles", "implementación de tecnologías móviles emergentes" y "desarrollo de habilidades a partir del mobile learning".

Nota: Elaboración propia, a partir del programa de análisis estadístico AMOS.

Atendiendo los resultados, se encuentran diferencias entre las respuestas de los futuros maestros y futuras maestras respecto a la inclusión de la metodología mobile learning en las aulas de educación primaria.

En general, con base en la escala Likert de 1 a 4, todas las medias se sitúan en valores por encima del 2, de modo que son positivas la mayoría de respuestas. Las únicas excepciones son el I3, I12 y I19, donde la mediana y moda se encuentran en el valor 2, correspondiente al nivel "en desacuerdo".

Centrando la atención en los porcentajes de respuesta, la mayor parte del estudiantado considera necesario el hecho de traer tu propio dispositivo al aula (BYOD), para que se puede implementar realmente una metodología basada en los dispositivos móviles (Suárez-Guerrero 
et al., 2016). Además, también remarcan la ubicuidad como una de sus principales ventajas, que convierten al dispositivo móvil en un potente recurso para el aprendizaje (Vázquez-Cano, 2015).

No obstante, en su mayoría consideran los dispositivos móviles como distractores (Cantillo et al., 2012; Gértrudix y Barroso-Megías, 2016), aunque opinan, en un porcentaje superior a la media, que su incorporación en el aula puede disminuir su uso inadecuado.

En relación con las tecnologías móviles emergentes, aprueban mayormente el uso de códigos QR para el aprendizaje en el aula (Romero y Rodríguez-García, 2017). También creen adecuada la implementación de la realidad aumentada como recurso educativo (Cabero et al., 2017). La realidad virtual y la impresión 3D muestran una mayor dispersión en las respuestas; sin embargo, la mayoría se muestra a favor de introducir actividades con realidad virtual, debido a sus amplias posibilidades (Aznar-Díaz et al., 2018). Al igual que la impresión 3D, es percibida como un recurso adecuado para el aprendizaje en estas edades (Saorin et al. 2016).

Los beneficios que traen consigo los dispositivos móviles quedan reflejados en la dimensión de desarrollo de habilidades, donde la mayoría del estudiantado considera que su introducción en el aula mejora la motivación (Cuesta y Mañas, 2016; Fombona y Pascual, 2013). En cambio, la mayor parte no cree que su uso favorezca el trabajo cooperativo, en contradicción con los trabajos de Monguillot et al. (2014) y Nguyen et al. (2015). En esta misma dimensión, si se refleja la posición favorable a un aumento de la participación a través del uso de plataformas virtuales o redes sociales.

Es interesante conocer que la mayor parte de los futuros maestros y maestras consideran que su introducción en esta etapa educativa puede ser una buena medida para evitar el ciberbullying. Del mismo modo que destacan la relación del dispositivo móvil con el desarrollo de competencias digitales, factor clave para preparar al alumnado a desenvolverse eficazmente en la sociedad actual (Gómez-Gonzalvo et al., 2015).

Cabe resaltar la opinión generalizada sobre la necesidad de combinar el mobile learning con otras metodologías como la clase magistral, es decir, no limitar la enseñanza únicamente al uso de los dispositivos móviles. En este sentido, es favorable intercalar distintos recursos educativos, puesto que es una opción de preferencia por parte del alumnado (Chaves-Barboza y Sola-Martínez, 2018).

Siguiendo con el análisis, casi la totalidad de los futuros maestros y maestras coinciden en la necesidad de que el personal docente se encuentre bien formado y posea conocimientos tecnológicos, que aseguren una correcta implementación de los dispositivos móviles.

Respecto a la introducción generalizada del mobile learning, es curioso el hecho de que la mayoría considere que no se implementará a corto plazo, en contra de los pronósticos del Informe Horizon (Adams et al., 2017). 
doi: http://dx.doi.org/10.15359/ree.23-3.14

URL: http://www.una.ac.cr/educare

CORREO: educare@una.cr

En suma, la mayoría de docentes aplicaría la metodología mobile learning en sus aulas, coincidiendo con la opinión positiva recogida en estudios similares (Contreras, 2010; Garay et al., 2016; Grané et al., 2013; Rivera et al., 2013). Por tanto, se percibe, entre el estudiantado, que los dispositivos móviles mejoran el aprendizaje (Wu et al., 2012).

Por último, el modelo de ecuaciones estructurales (Ruiz et al., 2010) nos permite conocer el grado de correlación entre los distintos ítems de las principales dimensiones establecidas. Así, se observa que a mayor percepción positiva en el uso de los dispositivos móviles en el aula, aumenta la creencia sobre el desarrollo de habilidades a partir de su implementación y la adecuación de aplicar tecnologías emergentes como la realidad aumentada, realidad virtual y la impresión 3D. Todo ello repercute en la aceptación de los dispositivos móviles y su posible inclusión en el futuro, como muestra la correlación perfecta entre la dimensión desarrollo de habilidades y el ítem 20.

\section{Conclusiones}

De acuerdo con el objetivo planteado acerca de conocer la opinión del futuro personal docente de educación primaria sobre la implementación de la metodología mobile learning en el aula, los resultados recogen una panorámica general que da respuesta a mencionado objetivo.

Así pues, el avance tecnológico es una constante en el mundo educativo, donde la tendencia es implementar estos recursos para la mejora del proceso de enseñanza-aprendizaje. Es primordial formar a los futuros maestros y a las futuras maestras en el buen uso de las TIC durante los estudios universitarios, ya que como indica el estudiantado, es necesaria la formación tecnológica del profesorado para una correcta aplicación de los dispositivos móviles. Así pues, el desarrollo de la competencia digital es un reto al que debemos hacer frente en la docencia universitaria. En este sentido, el personal docente universitario debe plantar la semilla tecnológica en sus programaciones didácticas para que florezca en sus estudiantes, quienes serán maestros y maestras de educación primaria en un futuro.

Este tipo de estudios nos muestra la visión de los maestros y las maestras del mañana, una opinión necesaria para conocer el grado de aceptación y conocimiento sobre metodologías emergentes como el mobile learning. Por tanto, es de interés para la comunidad científica contar con este tipo de investigaciones, que sacan a la luz diversas cuestiones clave en la puesta en práctica de los dispositivos móviles en las aulas.

Aunque algunos informes instan a la introducción del mobile learning a corto plazo, esta sensación no se ve reflejada en el día a día, ni en los futuros maestros y maestras. Por lo que su aplicabilidad generalizada seguramente ocurra en un periodo temporal a medio plazo, superior a la estimación de 1-2 años que indica el informe Horizon. 
Finalmente, como recomendaciones e implicaciones de los hallazgos encontrados destacan:

- Seguir investigando sobre la opinión del futuro personal docente, ya que el día de mañana serán quienes tengan que aplicar este tipo de metodologías innovadoras.

- El mobile learning y los dispositivos móviles se posibilitan como un potente recurso para la mejora del aprendizaje.

- Las tecnologías móviles emergentes (realidad aumentada y realidad virtual) están teniendo una buena aceptación por parte de los maestros y maestras, ya que implican un aprendizaje inmersivo y experiencial.

- Aún hay presentes ciertas reticencias a la aplicación de los dispositivos móviles en el aula por una parte de maestros y maestras.

- En unos años nos encontraremos con un escenario donde la cotidianeidad será el uso de las tecnologías móviles en la docencia.

\section{Referencias}

Adams, S., Cummins, M., Davis, A., Freeman, A., Hall, C. y Ananthanarayanan, V. (2017). NMC horizon report:2017 higher education edition. Austin, Texas: The New Media Consortium. Recuperado de https://www.sconul.ac.uk/sites/default/files/documents/2017-nmc-horizon-report-he-EN.pdf

Aznar-Díaz, l., Cáceres-Reche, M. P. y Romero-Rodríguez, J. M. (2018). Indicadores de calidad para evaluar buenas prácticas docentes de "mobile learning" en educación superior. Education in the Knowledge Society (EKS), 19(3), 53-68. doi: https://doi.org/10.14201/eks20181935368

Aznar-Díaz, I., Romero-Rodríguez, J. M. y Rodríguez-García, A. M. (2018). La tecnología móvil de realidad virtual en educación: Una revisión del estado de la literatura científica en España. edmetic, Revista de Educación Mediática y TIC, 7(1), 256-274. doi: https://doi.org/10.21071/ edmetic.v7i1.10139

Brazuelo, F. y Gallego, D. J. (2011). Mobile learning. Los dispositivos móviles como recurso educativo. Sevilla: MAD.

Cabero, J., Fernández, B. y Marín, V. (2017). Dispositivos móviles y realidad aumentada en el aprendizaje del alumnado universitario. RIED. Revista Iberoamericana de Educación a Distancia, 20(2), 167-185. doi: https://doi.org/10.5944/ried.20.2.17245

Cantillo, C., Roura, M. y Sánchez, A. (2012). Tendencias actuales en el uso de dispositivos móviles en educación. La Educ@cion Digital Magazine, 147, 1-21. Recuperado de http://educoas. org/portal/la educacion digital/147/pdf/art unned en.pdf 
doi: http://dx.doi.org/10.15359/ree.23-3.14

URL: http://www.una.ac.cr/educare

CORREO: educare@una.cr

Chaves-Barboza, E., y Sola-Martínez, T. (2018). Entornos personales de aprendizaje (PLE) en el grado de educación primaria de la Universidad de Granada. Revista Electrónica Educare, 22(1), 1-18. doi: https://doi.org/10.15359/ree.22-1.12

Contreras, R. S. (2010). Percepciones de estudiantes sobre el aprendizaje móvil; la nueva generación de la educación a distancia. Cuadernos de Documentación Multimedia, 21, 159-173. Recuperado de http://revistas.ucm.es/index.php/CDMU/article/view/ CDMU1010110159A/20733

Cuesta, U. y Mañas, L. (2016). Integración de la realidad virtual inmersiva en los grados de comunicación. Icono 14, 14(2), 1-21. doi: https://doi.org/10.7195/ri14.v14i2.953

Escobedo, M. T., Hernández, J. A., Estebané, V. y Martínez, G. (2016). Modelos de ecuaciones estructurales: Características, fases, construcción, aplicación y resultados. Ciencia \& Trabajo, 18(55), 16-22. doi: http://dx.doi.org/10.4067/S0718-24492016000100004

Fombona, J. y Pascual, M. A. (2013). Beneficios del m-learning en la educación superior. Educatio Siglo XXI, 31(2), 211-234. Recuperado de https://revistas.um.es/educatio/article/ view/187171/154271

Garay, U., Tejada, E. y Castaño, C. (2016). Percepciones del alumnado hacia el aprendizaje mediante objetos educativos enriquecidos con realidad aumentada. edmetic, Revista de Educación Mediática y TIC, 6(1), 145-164. Recuperado de https://doi.org/10.21071/edmetic.v6i1.5812

Gértrudix, F. y Barroso-Megías, M. (2016). ¿Es posible integrar los dispositivos móviles en educación primaria como recurso educativo? En J. Gómez-Galán, E. López-Meneses, A. H. Martín (Eds.), Advances and innovations in educational research (pp. 35-43). San Juan, Puerto Rico: UMET. Recuperado de https://dialnet.unirioja.es/servlet/libro?codigo=700047

Gómez-Gonzalvo, F., Atienza, R. y Mir, M. (2015). Revisión bibliográfica sobre usos pedagógicos de los códigos QR. @tic, revista d'innovació educativa, 15, 29-38. Recuperado de https:// dialnet.unirioja.es/servlet/articulo?codigo $=5300227$

Grané, M., Crescenzi, L. y Olmedo, K. (2013). Cambios en el uso y la concepción de las TIC, implementando el Mobile Learning. Revista de Educación a Distancia, 37, 1-19. Recuperado de https://revistas.um.es/red/article/view/234051/179821

Hernández, R., Fernández, C. y Baptista, P. (2016). Metodología de la investigación (6ª ed.). México: McGraw-Hill.

Monguillot, M., González, C., Guitert, M. y Zurita, C. (2014). Mobile learning: Una experiencia colaborativa mediante códigos QR. RUSCRevistade Universidad y Sociedad del Conocimiento, 11(1), 175-191. doi: http://dx.doi.org/10.7238/rusc.v11i1.1899 
Nguyen, L., Barton, S. M. y Nguyen, L.T. (2015). IPads in higher education - Hype and hope. British Journal of Educational Technology, 46(1), 190-203. doi: https://doi.org/10.1111/bjet.12137

Rivera, P., Sánchez, P., Romo, E., Jaramillo, A.y Valencia, A. (2013). Percepciones de los estudiantes universitarios frente al aprendizaje por medio de dispositivos móviles. Revista Educación y Desarrollo Social, 7(2), 152-165. doi: https://doi.org/10.18359/reds.687

Romero, J. M. y Rodríguez-García, A.-M. (2017). Digitalización de recursos patrimoniales mediante códigos QR: los dispositivos digitales móviles para la difusión cultural y artística en educación. En L. Bocanegra y A. García (Eds.), Con la red/en la red. Creación, investigación y comunicación cultural y artística en la era internet (pp. 595-612). New York, NY: Downhill Publishing. Recuperado de https://www.academia.edu/35518795/Con la Red En la Red. Creaci\%C3\%B3n Investigaci\%C3\%B3n y Comunicaci\%C3\%B3n Cultural y Art\%C3\%ADstica en la era Internet

Ruiz, M. A., Pardo, A. y San Martín, R. (2010). Modelos de ecuaciones estructurales. Papeles del psicólogo, 31(1), 34-45. Recuperado de http://www.papelesdelpsicologo.es/pdf/1794.pdf

Saorin, J. L., Meier, C., Ruiz, C., De la Torre-Cantrol, J., Melián, D., y Bonnet, A. (2016). Creación, visualización e impresión 3D de colecciones online de modelos educativos tridimensionales con tecnologías de bajo coste; caso práctico del patrimonio fósil marino de Canarias. Education in the Knowledge Society (EKS), 17(3), 89-108. doi: http://dx.doi.org/10.14201/ eks201617389108

Suárez-Guerrero, C., Lloret-Catalá, C. y Mengual-Andrés, S. (2016). Percepción docente sobre la transformación digital del aula a través de tabletas: Un estudio en el contexto español. Comunicar, 24(49), 81-89. doi: https://doi.org/10.3916/C49-2016-08

Vázquez-Cano, E. (2015). El reto de la formación docente para el uso de dispositivos digitales móviles en la educación superior. Perspectiva Educacional. Formación de Profesores, 54(1), 149-162. Doi: https://doi.org/10.4151/07189729-Vol.54-Iss.1-Art.236

Wu, W.-H., Wu, Y.-C. J., Chen, C.-Y., Kao, H.-Y., Lin, C.-H. y Huang, S.-H. (2012). Review of trends from mobile learning studies: A meta-analysis. Computers \& Education, 59(2), 817-827. doi: https://doi.org/10.1016/j.compedu.2012.03.016 